\title{
CLINICOPATHOLOGICAL AND IMMUNOHISTOCHEMICAL PROFILE OF ENDOCERVICAL ADENOCARCINOMA
}

\author{
Arockiasamy Babiya Infant ${ }^{1}$, Arunachalam Arputham²
}

${ }^{1}$ Assistant Professor, Department of Pathology, Thanjavur Medical College, Thanjavur.

${ }^{2}$ Assistant Professor, Department of Pathology, Thanjavur Medical College, Thanjavur.

ABSTRACT
BACKGROUND
Primary adenocarcinoma of cervix constitutes $10-15 \%$ of all cases of carcinoma of cervix, which is the second most common
carcinoma next to squamous cell carcinoma. Endocervical adenocarcinoma have a considerable morphological overlap with
endometrial adenocarcinoma though they differ in their aetiologies, behaviour, and treatments. This makes their diagnosis very
difficult particularly in biopsy or curetting specimens or when a fractional dilation and curettage specimens show adenocarcinoma
in both components of it. This study was done in the aim to suggest the possible origin of the tumour with the help of
immunohistochemistry.

\section{AIMS AND OBJECTIVES}

To identify the incidence, distribution, clinicopathological, histomorphological features of endocervical adenocarcinomas and to determine the immunohistochemical expression of CEA, Vimentin, ER and PR in endometrioid type of adenocarcinoma detected in endocervical biopsies, fractional dilation and curettage specimens (Both the components showing similar morphology), and in hysterectomy specimens to suggest the site of origin of tumour.

\section{MATERIALS AND METHODS}

It is a retrospective descriptive study of cervical adenocarcinomas conducted in the Institute of Obstetrics and Gynaecology, Madras Medical College, Chennai for a period of 4 years during the period between 2009 November to 2013 October. The statistical analysis was performed using statistical package for social science software version 11.5 the clinicopathological profile of the tumour were calculated using Student t-test and chi-square test.

\section{OBSERVATION AND RESULTS}

Among the total 13499 cases received during the study period, 2489 were cervical malignancies comprising 148 adenocarcinoma. It includes 101 mucinous (Endocervical) type, 44 endometrioid type, 2 serous type, and 1 clear cell type. Among the 30 cases of endometrioid type, 16 cases showed definite immunophenotype of cervical origin, 9 cases of endometrial origin and in the remaining 4 cases origin could not be made out as they showed aberrant immunophenotype. Association between CEA and tumour grade and FIGO stage was found to be statistically significant.

\section{CONCLUSION}

Identifying the expression of CEA, Vimentin, ER and PR in cervical adenocarcinoma can help to identify the origin of the tumour and plan the treatment preoperatively.

\section{KEYWORDS}

Endocervical Adenocarcinoma-Immunohistochemical Profile-CEA-VIM-ER-PR-Endometrioid Type.

HOW TO CITE THIS ARTICLE: Infant AB, Arputham A. Clinicopathological and immunohistochemical profile of endocervical adenocarcinoma. J. Evolution Med. Dent. Sci. 2016;5(54):3592-3598, DOI: 10.14260/jemds/2016/828

\section{INTRODUCTION}

Primary adenocarcinoma of cervix constitutes $10-15 \%$ of all cases of carcinoma of cervix. It is the second most common next to squamous cell carcinoma ${ }^{1,2}$ comprising a large heterogeneous group of neoplasms that exhibits a variety of histological patterns.

Endocervical adenocarcinoma and endometrial adenocarcinoma are two distinct entities with different

Financial or Other, Competing Interest: None.

Submission 02-06-2016, Peer Review 24-06-2016,

Acceptance 02-07-2016, Published 05-07-2016.

Corresponding Author:

Dr. Arockiasamy Babiya Infant,

Assistant Professor,

Department of Pathology,

Thanjavur Medical College,

Thanjavur.

E-mail:drbabi6@gmail.com

DOI: $10.14260 /$ jemds $/ 2016 / 828$ aetiologies, behaviour, and treatments, but there is considerable overlap in the morphological features of adenocarcinoma arising from these two different sites. This morphological overlap makes their histological differentiation, a diagnostic problem in biopsy or curetting specimens, especially in a small biopsy, or when a fractional dilation and curettage specimens show adenocarcinoma in both components of it. ${ }^{3}$

The preoperative distinction between these two is very important because the treatment for an endometrial cancer is commonly a simple hysterectomy (Sometimes, if the cervical involvement is identified before surgery, the treatment is modified radical hysterectomy), while for an endocervical adenocarcinoma primary chemotherapy is given with radical hysterectomy. Sometimes, even a combination of clinical, hysteroscopic, radiologic, and pathologic examination fails to identify the definite primary site. 
Several studies performed in the past suggested a panel of markers to differentiate these tumours. So, 30 cases of endometrioid type of adenocarcinoma were selected and subjected to a panel of 4 immunohistochemical markers, Carcinoembryonic Antigen (CEA), Vimentin (VIM), Oestrogenic Receptor (ER), and Progesteronic Receptor (PR).49 This study was done in the aim to suggest the possible origin of the tumour (Endocervix or endometrium) and to assess the clinicopathological profile of endocervical adenocarcinoma.

\section{MATERIALS AND METHODS}

This study is a retrospective descriptive study of endocervical adenocarcinomas conducted in a tertiary care hospital for a period of 4 years between 2009 November to 2013 October. Total of 13499 cases were received for histopathological examination in the Institute of Obstetrics and Gynaecology, Madras Medical College. Among 2489 cervical malignancies, 148 were adenocarcinoma, which constituted 5.94\%. In these 148 cases of adenocarcinoma of cervix, 118 were detected by endocervical biopsy, 15 were detected by hysterectomy, 15 were detected by fractional dilation and curettage specimens (Which showed similar features of malignancy in both the components). All the cases of adenocarcinomas irrespective of the age, stage were included for the study. Adenocarcinoma confined to uterine corpus and other morphological types of carcinoma of cervix other than adenocarcinoma were excluded.

Detailed history of the cases regarding age, history, type of procedure, site, stage, and histological type were obtained for all the 148 cases from pathology records. Haematoxylin and Eosin-stained $4 \mu$ thick sections of the paraffin tissue blocks of specimens were reviewed. Of the 148 cases, a total of 30 cases were selected and their representative formalin fixed paraffin embedded tissue samples were subjected to immunohistochemical analysis for a panel of 4 markers-CEA, Vimentin, ER and PR.

The immunohistochemically-stained slides were analysed for the presence of cellular localization, percentage of cells stained, and intensity of reaction. Cytoplasmic and membrane staining was noted for CEA, Typical paranuclear membrane, or lateral cell border staining was assessed for Vimentin ${ }^{6}$ and Nuclear staining for ER and PR.10

\begin{tabular}{|c|c|}
\hline $\begin{array}{l}\text { Negative } \\
\text { Staining }\end{array}$ & $\begin{array}{l}<1 \% \text { tumour cells show positive } \\
\text { staining }\end{array}$ \\
\hline $\begin{array}{l}\text { Weak Positive } \\
\text { Staining }\end{array}$ & $\begin{array}{l}1-10 \% \text { of tumour cells show weak } \\
\text { positive staining }\end{array}$ \\
\hline $\begin{array}{l}\text { Strong Positive } \\
\text { Staining }\end{array}$ & $\begin{array}{l}>10 \% \text { of tumour cells show positive } \\
\text { staining }\end{array}$ \\
\hline $\begin{array}{r}\text { Table 1: Criter } \\
\text { Percento }\end{array}$ & $\begin{array}{l}\text { Immunohistochemical Analysis of } \\
\text { Cells }^{6} \text { Expressing the Marker }\end{array}$ \\
\hline
\end{tabular}

This table illustrates the criteria for immunohistochemical analysis of percentage of cells ${ }^{6}$ expressing the marker.

Simplified scoring results then were made as either negative or positive results (Combining weak and strong positive results), unsatisfactory/uninterpretable results were discarded from further consideration.

The statistical analysis was performed using statistical package for social science software version 11.5, which consisted computing the frequency counts and percentages for qualitative variables and mean for the quantitative variables.
The clinicopathological profile of the tumour were calculated using Student t-test and chi-square s test.

\section{STATISTICS AND RESULTS}

Among 2489 cervical malignancies, 148 were adenocarcinoma, which includes 101 of mucinous type, 44 of endometrioid type, 2 of serous type, and 1 clear cell type. Adenocarcinoma of cervix constituted for $5.94 \%$ of cervical malignancies received and endometrioid type constituted $29.73 \%$ of adenocarcinoma.

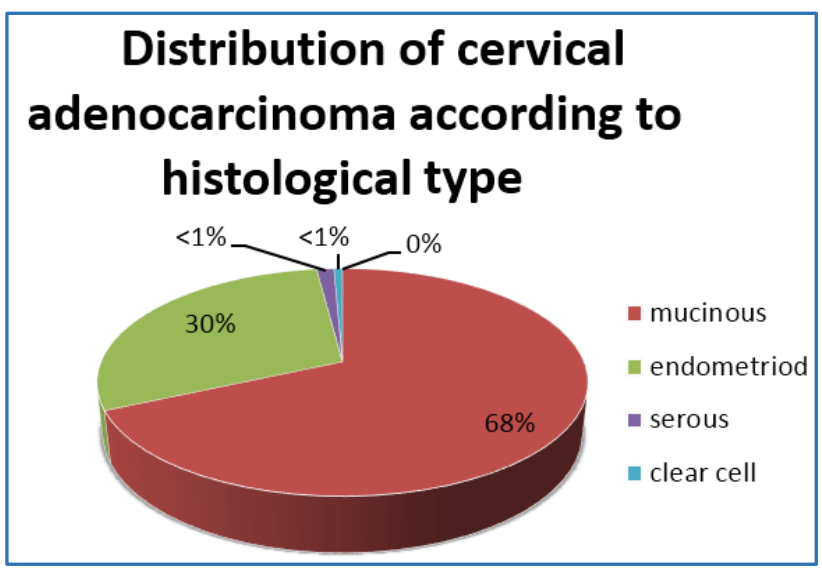

Adenocarcinoma of cervix showed a peak incidence in the age group of 40 to 50 years. The oldest age of presentation was 81 years and youngest age of presentation was 22 years. Mean age at presentation is 50.20 years.

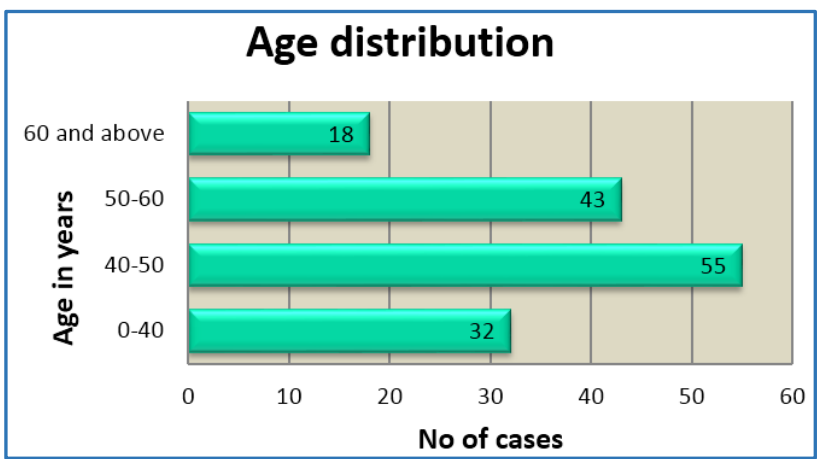

The distribution of endocervical adenocarcinoma based on histological grading showed majority of well differentiation. (94 cases-63\%) followed by moderate differentiation (29 cases-19.59\%). 84 cases presented in the FIGO stage of II (56.75\%), followed by stage I (51-34.45\%), and stage III and IV $(8.7 \%)$.

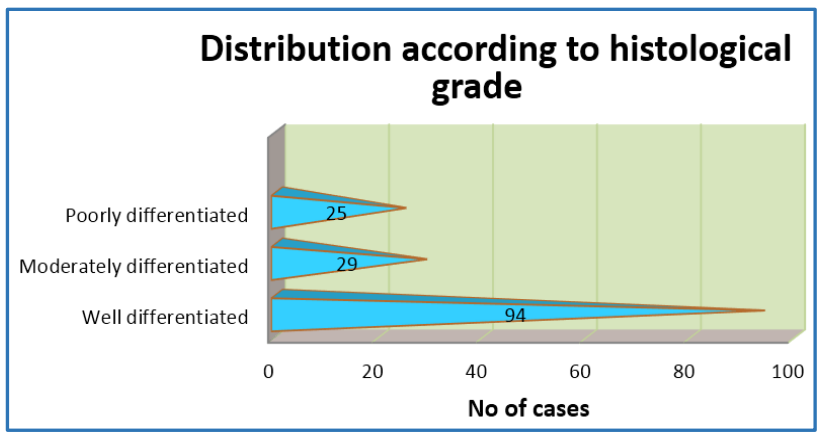




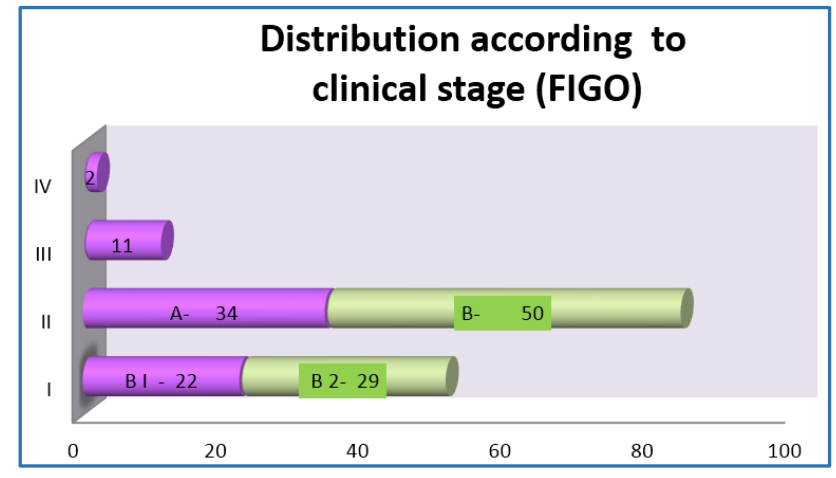

Among the 30 cases of endometrioid type, 16 cases showed definite immune phenotype of endocervical origin [i.e., a combination of CEA positivity, VIM negativity, ER negativity] and 9 cases showed definite immunophenotype of endometrial origin [i.e., a combination of CEA negativity, VIM positivity, ER positivity] and in the remaining 4 cases origin could not be made out as they showed aberrant immunophenotype.

Among the 17/30 cases, which showed CEA positivity, 16 cases show concomitant negative expression of VIM, ER suggesting endocervical origin.

Expression of CEA in Comparison with Other Markers

\begin{tabular}{|c|c|c|c|c|c|c|}
\hline & VIM+ & VIM- & ER+ & ER- & PR+ & PR- \\
\hline CEA+ & 1 & 16 & 1 & 16 & 2 & 15 \\
\hline CEA- & 10 & 3 & 9 & 4 & 6 & 7 \\
\hline
\end{tabular}

Among the 11/30 cases, which showed VIM positivity, 9 cases show concomitant negative expression of CEA and positive expression of ER suggesting endometrial origin.

\section{Expression of Vimentin in Comparison with Other} Markers

\begin{tabular}{|c|c|c|c|c|c|c|}
\hline & CEA+ & CEA- & ER+ & ER- & PR+ & PR- \\
\hline VIM+ & 1 & 10 & 10 & 1 & 6 & 5 \\
\hline VIM- & 16 & 3 & 0 & 19 & 1 & 18 \\
\hline
\end{tabular}

Correlation of CEA with Various Clinicopathological Factors like Age, Grade, Stage of Tumour

The mean age of patients with CEA expression was 49.29 years and that of patients without CEA expression was 53.69 years. There was no significant difference in the age at presentation between the two groups.

Among the 17 cases of CEA positivity, 11 cases showed moderate differentiation. Significant association was found between the CEA expression and tumour grade as depicted in Table 2 .

\begin{tabular}{|c|c|c|c|}
\hline Grade & Positive & Negative & Total \\
\hline Well Differentiated & 3 & 11 & 14 \\
\hline Moderately Differentiated & 11 & 1 & 12 \\
\hline Poorly Differentiated & 3 & 1 & 4 \\
\hline Total & $\mathbf{1 7}$ & $\mathbf{1 3}$ & $\mathbf{3 0}$ \\
\hline Chi-Square Test & \multicolumn{3}{|c|}{ p value 0.001 } \\
\hline $\begin{array}{r}\text { Table 2: Association of Tumour Grade } \\
\text { with CEA Expression }\end{array}$ \\
\hline
\end{tabular}

Of the 17 CEA positive cases, 12 cases presented in stage IIB. Significant association was found between stage and CEATable 3.

\begin{tabular}{|c|c|c|c|}
\hline Stage & Positive & Negative & Total \\
\hline IB1 & 1 & 6 & 7 \\
\hline IB2 & 1 & 3 & 4 \\
\hline IIA & 1 & 2 & 3 \\
\hline II B & 12 & 1 & 13 \\
\hline III & 2 & 1 & 3 \\
\hline IV & 0 & 0 & 0 \\
\hline Total & 17 & 13 & $\mathbf{3 0}$ \\
\hline Chi-square test & \multicolumn{3}{|c|}{ p value 0.006 } \\
\hline \multicolumn{3}{|c|}{ Table 3: Association of Tumour } \\
\hline
\end{tabular}

\begin{tabular}{|c|c|c|}
\hline \multicolumn{2}{|c|}{ Clinicopathological Factor } & No. of Cases (\%) \\
\hline \multirow[b]{2}{*}{ Age } & $<50$ Years & $87(58.7 \%)$ \\
\hline & $>50$ Years & $61(41.2 \%)$ \\
\hline \multirow{4}{*}{ Histological Type } & Mucinous & $101(68.2 \%)$ \\
\hline & Endometrioid & $44(29.7 \%)$ \\
\hline & Serous & $2(1.35 \%)$ \\
\hline & Clear Cell & $1(0.67 \%)$ \\
\hline \multirow{3}{*}{ Grade } & I & $94(63.5 \%)$ \\
\hline & II & $29(19.5 \%)$ \\
\hline & III & $25(16.8 \%)$ \\
\hline \multirow{4}{*}{ Stage } & $\mathrm{I}$ & $51(34.4 \%)$ \\
\hline & II & $84(56.7 \%)$ \\
\hline & III & $11(7.4 \%)$ \\
\hline & IV & $2(1.35 \%)$ \\
\hline \multirow{2}{*}{ CEA } & Positive & $17(56.67 \%)$ \\
\hline & Negative & $13(43.34 \%)$ \\
\hline \multirow{2}{*}{ VIM } & Positive & $11(36.67 \%)$ \\
\hline & Negative & $19(63.34 \%)$ \\
\hline \multirow{2}{*}{ ER } & Positive & $10(33.64 \%)$ \\
\hline & Negative & $20(66.67 \%)$ \\
\hline \multirow{2}{*}{ PR } & Positive & $7(23.34 \%)$ \\
\hline & Negative & $23(76.67 \%)$ \\
\hline \multicolumn{3}{|c|}{$\begin{array}{l}\text { Table 4: Overall Clinicopathological } \\
\text { Profile of Cervical Adenocarcinoma }\end{array}$} \\
\hline
\end{tabular}

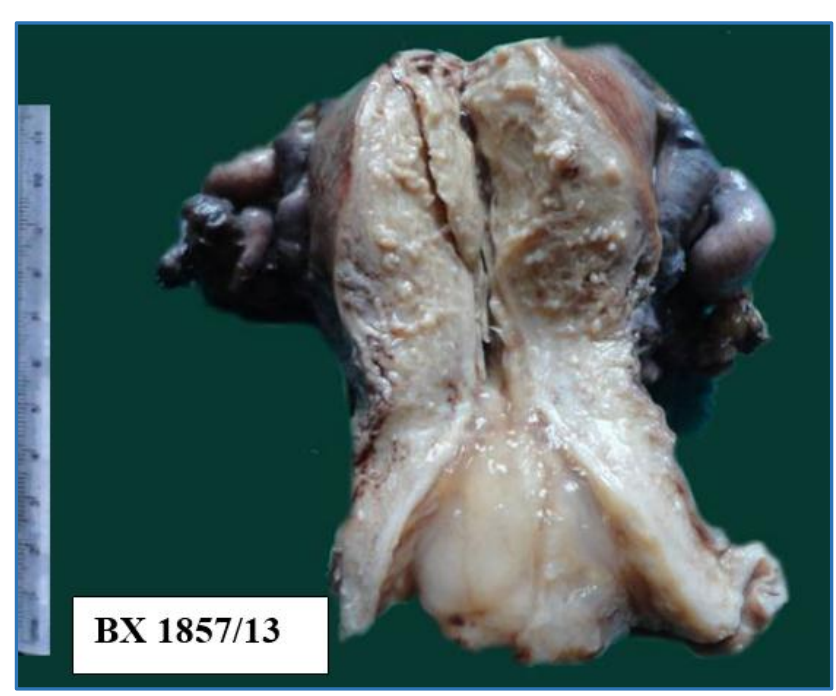

Fig. 1: Gross Picture of TAH Specimen with a Polypoidal Growth Arising from the Endocervix 


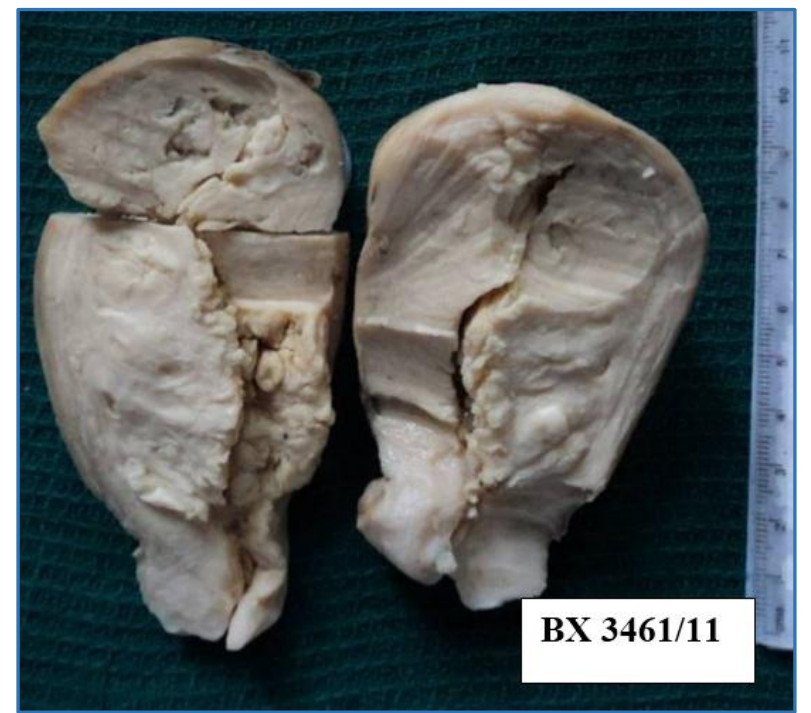

Fig. 2: Gross Picture of TAH Specimen Showing a Proliferative Growth Involving the Endocervix

Endometrioid Adenocarcinoma Arising from Endocervix

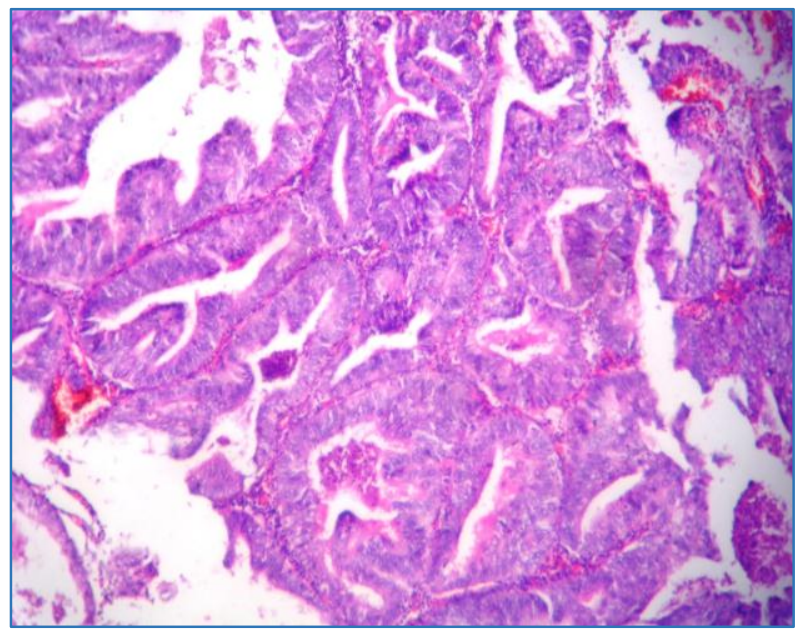

Fig. 3: Tubules and Glands of Various Sizes Arranged Back to Back, lined by Stratified Columnar Epithelium Exhibiting Mild Atypia, 10 X, HPE 1171/10

Immunohistochemical Analysis of Endometrioid Adenocarcinoma-Arising from Endocervix

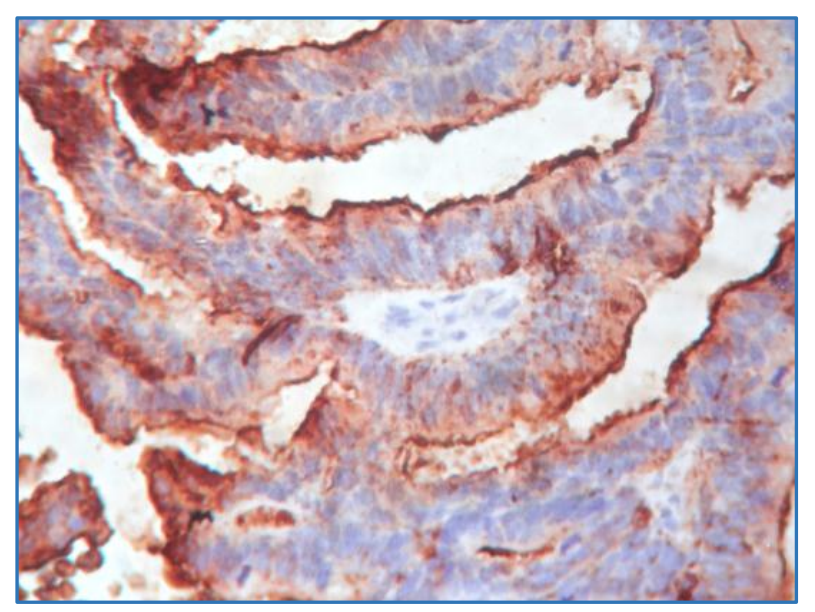

Fig. 4: CEA Staining, Malignant Glands Show Strong Cytoplasmic and Membrane Staining, $40 \mathrm{X}$, HPE 1171/10

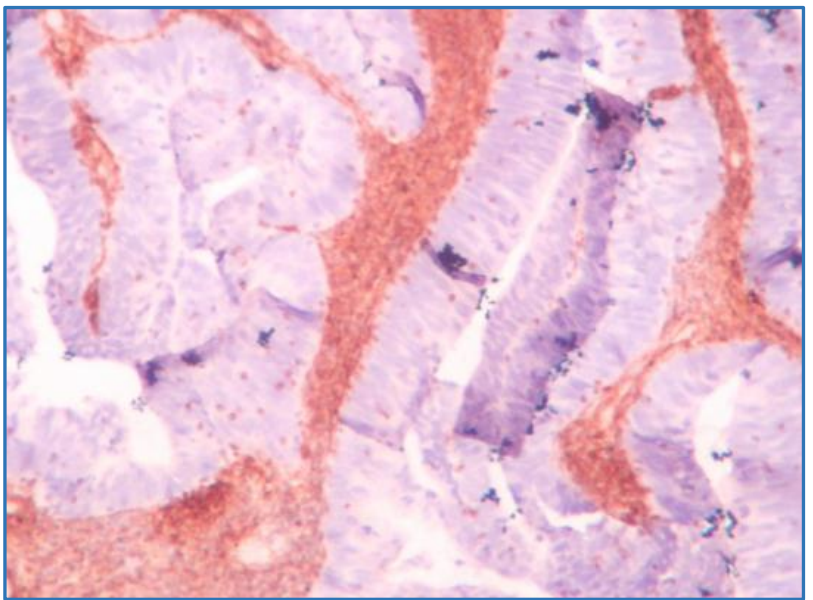

Fig. 5: VIM Staining, Malignant Glands Show Negative Staining, (Underlying Endometrial Stroma Show Positive Staining) $40 \mathrm{X}$, HPE 1171/10

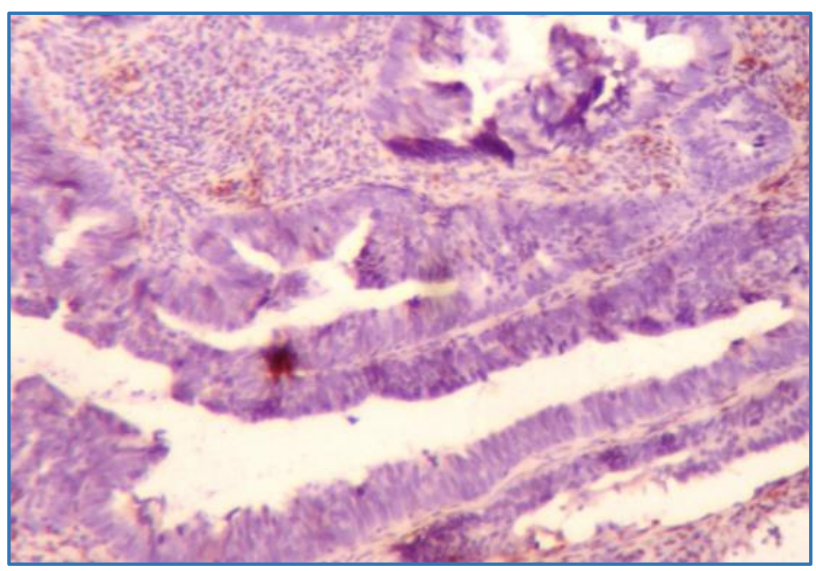

Fig. 6: ER Staining, Malignant Glands Show Negative Staining, (Underlying Endometrial Stroma Show Positive Staining) $10 \mathrm{X}$, HPE-1171/10

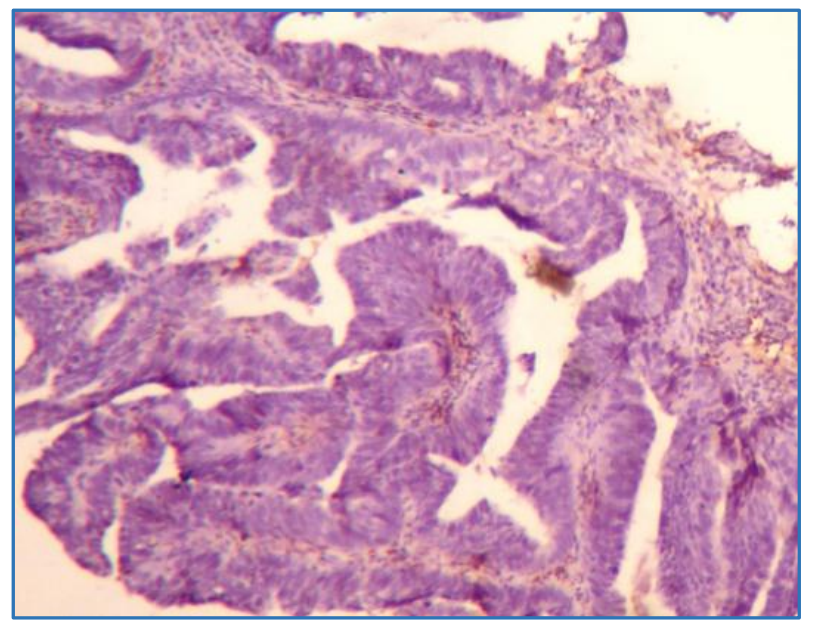

Fig. 7: PR Staining, Malignant Glands Show Negative Staining, (Underlying Endometrial Stroma Show Positive Staining) $10 \mathrm{X}$, HPE-1171/10 
Endometrioid Adenocarcinoma Arising from Endometrium

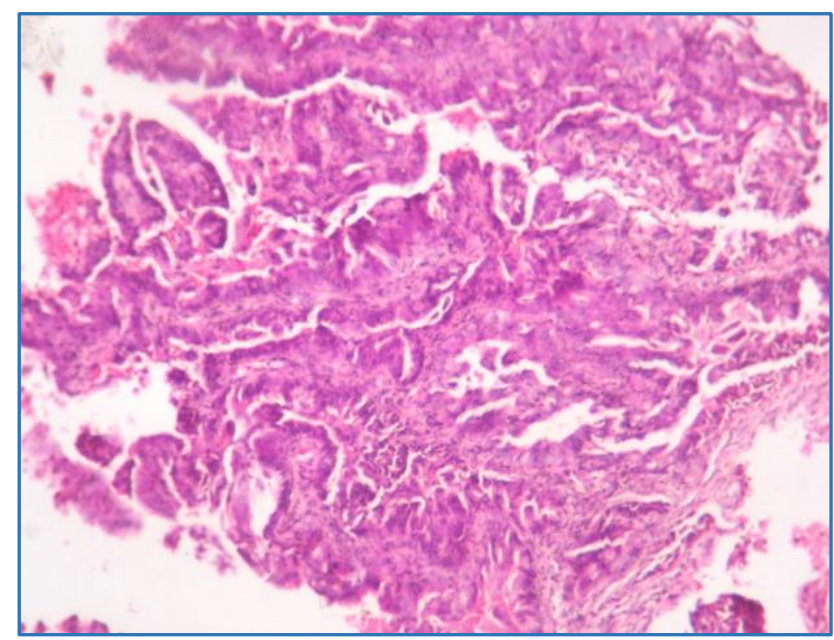

Fig. 8: Tubules and Glands Fused Together with Stromal Invasion Lined by Stratified Columnar Epithelium Exhibiting Moderate Atypia, 10 X, HPE- 7/11

Immunohistochemical Analysis of Endometrioid Adenocarcinoma - Arising from Endometrium

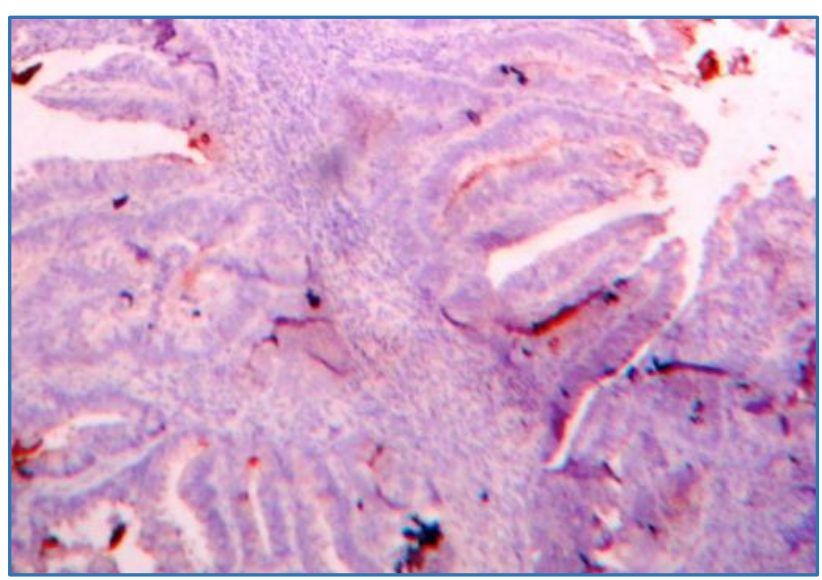

Fig. 9: CEA Staining, Malignant Glands Show Negative Staining, $10 \mathrm{X}, \mathrm{HPE}-7 / 11$

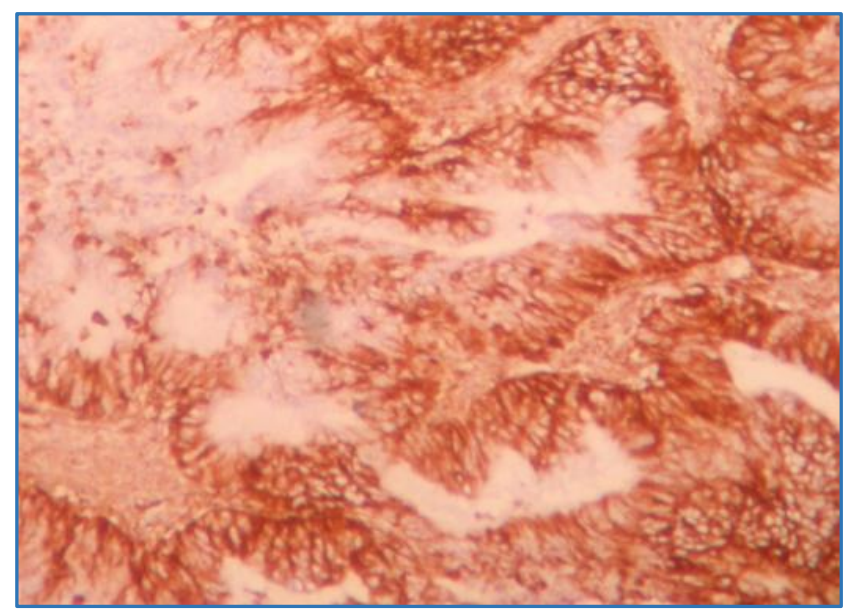

Fig. 10: VIM Staining, Malignant Glands Show Positive Cytoplasmic Staining, (Underlying Endometrial Stroma Show Positive Staining) 10 X, HPE 7/11

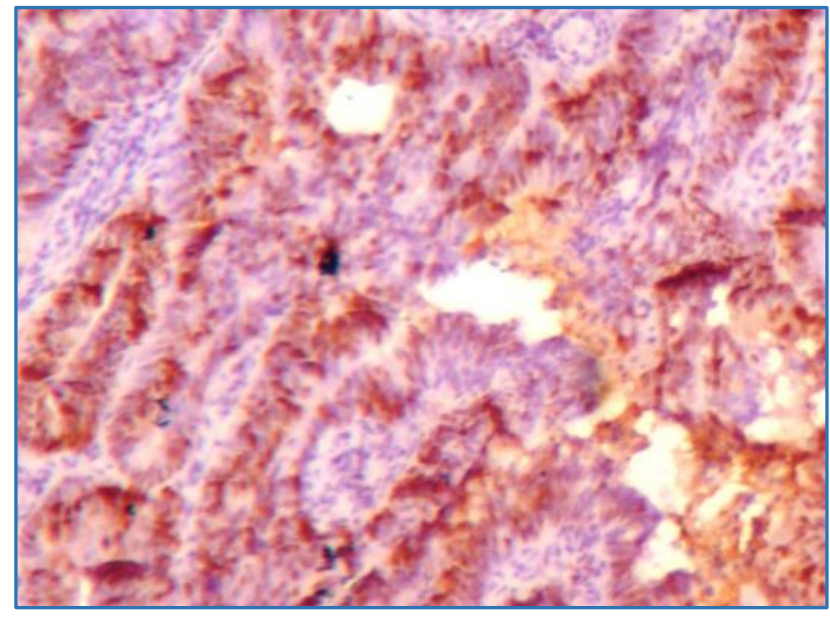

Fig. 11: ER Staining, Malignant Glands Show Strong Nuclear Staining, 10 X, HPE-7/11

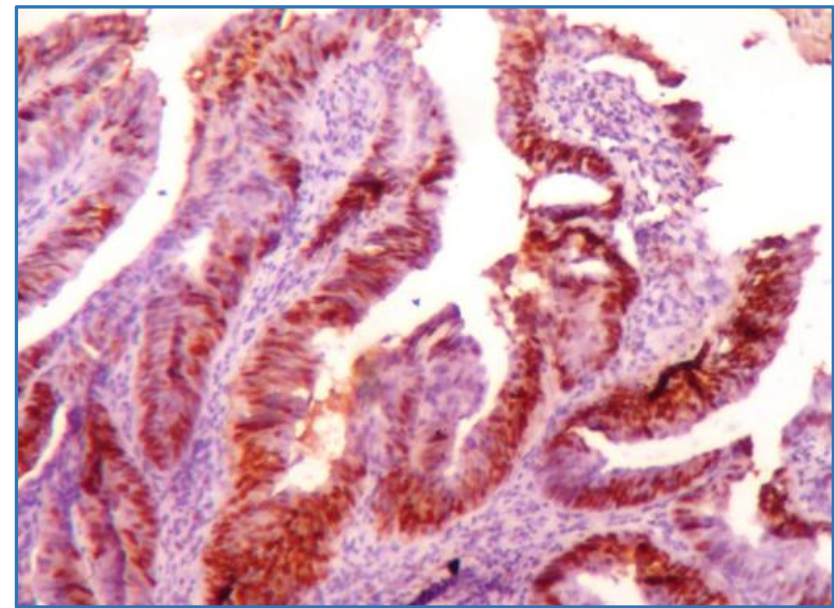

Fig. 12: PR Staining, Malignant Glands Show Strong Nuclear Staining, 10 X, HPE 7/11

\section{DISCUSSION}

Carcinoma of cervix is the third leading cause of death in females of age group 20-39. The age of diagnosis and death has come down. Recently estimated new incidence of cancer cases is 12,340 and deaths is 4,030 , in United States in 2013. The probability of developing uterine cervical cancer from birth to death is 0.68 ( 1 in 147). ${ }^{11}$

Cervical malignancy kills nearly 72,000 women every year in India, which is more than $26 \%$ of the 2,75,000 deaths happening all over the world. More than $50 \%$ of cervical cancer deaths happen altogether in India, China, Bangladesh, Nigeria, and Brazil among the worldwide countries. ${ }^{12}$

Endocervical adenocarcinoma and endometrial adenocarcinoma are two distinct entities. In spite of being different in their aetiologies, behaviour, and treatments, they have a considerable morphological overlap, which makes their diagnosis very difficult particularly in biopsy or curetting specimens especially in a small biopsy or when a fractional dilation and curettage specimens show adenocarcinoma in both components of it.

Adenocarcinoma of cervix showed a peak incidence in the age group of 40 to 50 years. Mean age at presentation is 50.20 years. This is in concurrence with the study conducted by Klaus Bodner et al (2010) ${ }^{13}$ who observed the median age of 51 years with the range of 24-60 years and the study conducted by Farveen Marican Abu Backer et al ${ }^{14}$ who 
observed the median age to be 48.5 years in the patients of cervical adenocarcinoma.

The common tumour subtype among adenocarcinoma detected in cervix in this study is mucinous type followed by endometrioid type, which is similar to the study by Fujiwara et al15 (1997), who observed mucinous (Endocervical type) to be most common $57 \%$ and the study conducted by Farveen Marican Abu Backer et al ${ }^{14}$ and Klaus Bodner et al (2010). ${ }^{13}$

In the present study, well differentiated tumours were more common accounting to $63.5 \%$. This was similar to the study conducted by Klaus Bodner et al (2010) ${ }^{13}$ and the study conducted by Farveen Marican Abu Backer et al ${ }^{14}$ in which the combination of well and moderately differentiated came to be $62.5 \%$ in the patients of cervical adenocarcinoma.

This study showed a higher proportion of stage II tumours followed by stage I tumours, which did not concur with the studies conducted Klaus Bodner et al (2010)13 and Farveen Marican Abu Backer et al $^{14}$ who showed a predominance of tumours of stage I in the patients of cervical adenocarcinoma.

Among the 30 cases of endometrioid type, 16 cases showed definite immunophenotype of endocervical origin [i.e., a combination of CEA positivity, VIM negativity, ER negativity] and 9 cases showed definite immunophenotype of endometrial origin [i.e., a combination of CEA negativity, VIM positivity, ER positivity] and in the remaining 4 cases origin could not be made out.

This impression was based on the study conducted by Castrillon et al (2002), 6 who observed CEA positivity in $62 \%$ of endocervical adenocarcinoma, but Vimentin positivity in only $7 \%$ of it. He also compared the CEA, VIM expression in endometrial adenocarcinoma, which came to be $27 \%$ and $100 \%$ respectively. Another study conducted by McCluggage et $\mathrm{al}(2002)^{7}$ showed CEA, VIM, ER expression in cervical adenocarcinoma to be $96 \%, 8 \%, 38 \%$ respectively, while CEA, VIM, ER expression in endometrial adenocarcinoma was found to be $70 \%, 97 \%, 93 \%$.

Similar study by A. Alkushi et al (2003) ${ }^{3}$ showed CEA, VIM, ER positivity in $70 \%, 11 \%, 11 \%$ of cervical adenocarcinoma respectively and CEA, VIM, ER positivity in $61 \%, 92 \%, 73 \%$ of endometrial adenocarcinoma.

\section{Limitations of this Study}

1. This study was conducted in a large tertiary hospital, so the findings therefore cannot be generalized to the whole community.

2. This panel of IHC markers is useful mainly in the distinction between endocervical adenocarcinoma and a low-grade endometrioid adenocarcinoma of the corpus. High-grade aggressive uterine adenocarcinoma do not express ER.

3. As the exact localisation of the tumour was not made out, the immunological suggestion of the origin of the tumour are not confirmed.

\section{Scope for Future Studies}

1. Analysis of HPV association in cervical adenocarcinomas helps to identify the primary, so the immunostains for $\mathrm{p} 16$ (INK4A) will be helpful in differentiating these two malignancies.

2. Exact preoperative localization of the tumour by various advanced techniques with proper follow up of the cases might be very useful to identify the role of the above markers as both diagnostic and prognostic factors.

\section{CONCLUSION}

The incidence of cervical adenocarcinoma for a period of 4 years in the Institute of Obstetrics and Gynaecology, Madras Medical College is $5.94 \%$ of total cervical malignancies with the peak incidence in the age group of 40 to 50 years and the most common histological type noted was mucinous type (Endocervical type) followed by endometrioid type with majority of the cases exhibiting well differentiation, which is similar to several other studies conducted throughout the world.

Among the 30 cases of endometrioid type, 16 cases showed definite immunophenotype of cervical origin and 9 cases showed definite immunophenotype of endometrial origin and in the remaining 4 cases origin could not be made out as they showed aberrant immunophenotype.

To conclude, identifying the expression of CEA, Vimentin, ER and PR in cervical adenocarcinoma can help to identify the origin of the tumour and plan the treatment preoperatively. Future studies with a larger sample size with exact localization of the tumour with proper follow up of the cases might shed more light on the role of the above markers in both diagnosis and treatment of the cervical adenocarcinoma.

\section{REFERENCES}

1. Smith HO, Tiffany MF, Qualls CR, et al. The rising incidence of adenocarcinoma relative to squamous cell carcinoma of the uterine cervix in the United States-a 24-year population-based study. Gynaecol Oncol 2000;78(2):97105.

2. Vizcaino AP, Moreno V, Bosch FX, et al. International trends in the incidence of cervical cancer: I adenocarcinoma and adenosquamous cell carcinomas. Int J Cancer 1998;75(4):536-45.

3. Alkushi A, Irving J, Hsu F, et al. Gilks immunoprofile of cervical and endometrial adenocarcinomas using a tissue microarray. Virchows Arch 2003;442(3):271-7.

4. McCluggage WG. Immunohistochemistry as a diagnostic aid in cervical pathology. Pathology 2007;39(1):97-111.

5. Kong CS, Beck AH, Longacre TA. A panel of 3 markers including p16, ProExC, or HPV ISH is optimal for distinguishing between primary endometrial and endocervical adenocarcinomas. Am J Surg Pathol 2010;34(7):915-26.

6. Castrillon DH, Lee KR, Nucci MR. Distinction between endometrial and endocervical adenocarcinoma: an immunohistochemical study. Int J Gynaecol Pathol 2002;21(1):4-10.

7. McCluggage WG, Sumathi VP, McBride HA, et al. A panel of immunohistochemical stains, including carcinoembryonic antigen, Vimentin, and oestrogen receptor, aids the distinction between primary endometrial and endocervical adenocarcinomas. Int J Gynaecol Pathol 2002;21(1):11-5.

8. Dabbs DJ, Geisinger KR, Norris HT. Intermediate filaments in endometrial and endocervical carcinomas the diagnostic utility of Vimentin patterns. Am J Surg Pathol 1986;10(8):568-76.

9. McCluggage WG, Jenkins D. P16 immunoreactivity may assist in the distinction between endometrial and endocervical adenocarcinoma. Int J Gynaecol Pathol 2003;22(3):231-5. 
10. Radu A, Frandes CD, Pop 0 , et al. The immunehistochemical study of endometrial and endocervical carcinomas using anti-Vimentin in co-expression with anti-ER. Studia Universitatis Vasile Goldiş, Seria Stiintele Vietii 2010;20(3):23-6.

11. Siegel R, Naishadham D, Jemal A. Cancer statistics 2013. CA Cancer J Clin 2013;63(1):11-30.

12. A chart CCFC compiled using data from the World Health Organization, the United Nations, the World Bank and the International Agency for Research on Cancer Globocan.

13. Bodner K, Laubichler P, Kimberger O, et al. Oestrogen and progesterone receptor expression in patients with adenocarcinoma of the uterine cervix and correlation with various clinicopathological parameters. Anticancer Research 2010;30(4):1341-5.
14. Backer FMA, Mustapha NRN, Othman NH. Clinicopathological comparison of adenocarcinoma of cervix and endometrium using cell cycle markers: p16ink4a, p21waf1, and p27Kip1 on 132 cancers. Infectious Diseases in Obstetrics and Gynaecology, Article ID 857851, 2011;2011:6.

15. Fujiwara H, Tortolero-Luna G, Mitchell MF, et al. Adenocarcinoma of the cervix. Expression and clinical significance of oestrogen and progesterone receptors. Cancer 1997;79(3):505-12. 